\title{
An update on belimumab for the treatment of lupus
}

This article was published in the following Dove Press journal:

Biologics:Targets and Therapy

II February 20II

Number of times this article has been viewed

\author{
Aikaterini Thanou-Stavraki ${ }^{1}$ \\ Amr H Sawalha ${ }^{1,2,3}$ \\ 'Department of Medicine, University \\ of Oklahoma Health Sciences \\ Center, Oklahoma City, OK, USA; \\ ${ }^{2}$ US Department of Veterans Affairs \\ Medical Center, Oklahoma City, \\ OK, USA; ${ }^{3}$ Arthritis and Clinical \\ Immunology Program, Oklahoma \\ Medical Research Foundation, \\ Oklahoma City, OK, USA
}

\begin{abstract}
B-lymphocyte stimulator (BLyS), a homeostatic factor for B-cell differentiation and survival, has a major role in B-cell expansion and autoreactivity that characterize systemic lupus erythematosus (SLE). Belimumab, a BLyS-specific inhibitor, has shown promising evidence of efficacy in several preclinical and clinical studies in SLE. Two recent large randomized controlled trials yielded a significant positive effect of the drug compared to placebo in patients with active disease. In this review, we discuss basic aspects of B-cell and BLyS biology in SLE and summarize the evidence supporting a role of belimumab in SLE, from animal studies to phase III clinical trials.
\end{abstract}

Keywords: B lymphocyte stimulator, lupus erythematosus, belimumab

\section{Introduction}

It has been more than 50 years since a new medication was approved by the Food and Drug Administration (FDA) for the treatment of lupus. ${ }^{1}$ Although several of the examined biologics have yielded promising results in open-label studies, none showed a considerable effect in randomized controlled trials (RCT), sufficient to pass the rigorous scientific FDA standards of approval. ${ }^{2}$ The genetic and phenotypic heterogeneity characteristic of systemic lupus erythematosus (SLE), the frequently undulating disease activity causing difficulties capturing a response, and the confounding effect of background immunosuppressive treatments are considered the main reasons for this failure. ${ }^{1}$ These notions were well illustrated in the two phase III rituximab trials in SLE that failed to show clinical benefit, despite wide preliminary evidence of efficacy. ${ }^{3}$

In this discouraging scene of biologics in SLE, hope has been raised by the modest yet promising results yielded by belimumab, a fully human recombinant immunoglobulin $\mathrm{G}$ ( $\mathrm{IgG}) 1 \lambda$ monoclonal antibody to soluble B-lymphocyte stimulator (BLyS). ${ }^{4}$ Belimumab is the first and only biologic to show consistent efficacy in two large phase III RCTs in SLE. Extension arms of these trials are ongoing with projected completion in December 2010, when a pending priority FDA review may grant the medication regulatory approval in the United States.

\section{B cells in SLE}

SLE, the prototypic autoimmune disease, is associated with numerous abnormalities in B-cell development, maturation, and function. ${ }^{5}$ Immune deregulations in SLE are characterized by loss of tolerance to ubiquitous self antigens and are propagated by multiple interactions between innate and adaptive immune pathways, culminating in 
end-organ damage. ${ }^{6}$ Immature transitional cells, memory cells, and plasma cells expand in the periphery, and autoreactive cells are incompletely deleted in the bone marrow of SLE patients. ${ }^{5}$ Overactive T-cell-dependent germinal center (GC)-like reactions produce expanded and largely unregulated numbers of memory B cells and plasma cells. ${ }^{5}$ Once tolerance is broken, autoreactive B cells can perpetuate disease, not only through autoantibody production and immune complex deposition, but also by multiple other effector functions. Such B-cell functions include production of cytokines, antigen presentation to $\mathrm{T}$ cells, induction of epitope spreading, and amplification of tissue-specific autoimmune responses. ${ }^{7}$

\section{Biology of BLyS}

BLyS, also known as B-cell-activating factor (BAFF), is a 285-amino acid cytokine with a central physiologic role in B-cell homeostasis and survival. ${ }^{8}$ BLyS and its closely related homologue APRIL (a proliferation-inducing ligand) are members of the tumor necrosis factor (TNF) superfamily expressed by a variety of cell types, mainly innate immune cells, like neutrophils, macrophages, monocytes, and dendritic cells, but also some T and B lymphocytes. ${ }^{9}$ Several other nonhematopoietic cells expressing these cytokines have been recently identified, possibly modulating B-cell functions in the tissue microenvironment. ${ }^{9}$ Both BLyS and APRIL are synthesized as membrane-spanning monomers and associate to form homotrimers that are proteolytically processed to soluble ligands. ${ }^{8,9}$ BLyS can be also found in a membrane-bound form. ${ }^{9}$

BLyS interacts with three different receptors: BR3 (BAFF receptor 3), also known as BAFF receptor, TACI (transmembrane activator and calcium modulator and cyclophylin ligand interactor), and BCMA (B-cell maturation antigen). ${ }^{9}$ BR3 binds solely to BLyS, whereas TACI and BCMA ligate both BLyS and APRIL. All three receptors signal through TNF receptor-associated factors (TRAFs), activating the nuclear factor-kB (NF-kB) and other pathways. ${ }^{9} \mathrm{BR} 3$ is a potent stimulator of the alternative NF-kB pathway, whereas TACI and BCMA stimulate the classic NF-kB pathway. Receptor specificities, affinities, and signal transduction pathways have been reviewed elsewhere. ${ }^{8,9}$ Variations in receptor-ligand affinities and distinct patterns and levels of receptor expression among different stages of B-cell development link them to a wide array of biological effects. BR3 expression is low in newly formed immature B cells, but progressively increases throughout B-cell development and is universal in mature B cells. ${ }^{8}$ TACI is also an inducible receptor, expressed by all peripheral
B cells. On the other hand, BCMA expression is restricted to antibody-producing cells. ${ }^{8}$ These receptors are also expressed in several other immune and nonimmune cell types. ${ }^{9}$

In the early stages of B-cell development, BLyS binding to BR3 provides an important survival signal by antagonizing apoptosis and allowing further differentiation and entrance of transitional cells into mature B-cell pools. ${ }^{8}$ BR3 expression, and thus the capacity to bind BLyS, rises in parallel with expression of the B-cell receptor (BCR). As basal BCR signaling is also required for positive selection of healthy B-cells and low-affinity autoreactive B cells, both pathways affect the stringency of naive B-cell selection in the periphery. ${ }^{8}$ Antigen-experienced B-cell subsets are however independent of the BLyS/BR3 interaction, relying on TACI and BCMA for maturation and differentiation signals. ${ }^{8}$ TACI is essential for efficient T-cell-independent type II humoral immune responses that yield antibody-producing plasmablasts and short-lived plasma cells. ${ }^{8}$ Although BLyS and APRIL are not required for generation of T-cell-dependent GCs, they play a role in appropriate evolution of the overall GC reactions that yield long-lived plasma cells and memory cells. TACI-APRIL interactions affect B-cell survival and class switching (CD 40 independent) to IgA during T-cell-dependent responses. ${ }^{9}$ Long-lived plasma cells in the bone marrow primarily express BCMA, which promotes their survival and function through ligation of APRIL or BLyS. ${ }^{8}$ In contrast, most evidence supports a lack of reliance of memory B cells on BLyS or APRIL for their survival or reactivation. ${ }^{8}$

\section{BLyS in SLE}

Lupus-prone mice have provided a valuable tool for studying the effects of BLyS excess and BLyS blockade to immunologic parameters and clinical manifestations of SLE. BLyS transgenic mice develop B-cell hyperplasia, hypergammaglobulinemia, autoantibodies, including antinuclear and anti-double-stranded DNA (anti-dsDNA) antibodies, and autoimmune symptoms reminiscent of SLE or Sjogren's syndrome. ${ }^{10}$ Conversely, application of selective or nonselective BLyS blockade in lupus-prone mice attenuates inflammation and even induces remission of nephritis in some strains. ${ }^{7}$ Selective blockade of BLyS in New Zealand black/white (NZB/W) F1 mice, the prototypical model of SLE nephritis, effectively depleted splenic B cells, sparing B1 and T1 cells. ${ }^{7}$ Although spontaneous GCs still formed and pathogenic autoantibodies were produced in these mice, long-lasting effects on splenic architecture and on B-cell-dependent accumulation of $\mathrm{T}$ cells and dendritic cells were observed. ${ }^{7}$ BLyS-specific blockade in NZB/W F1, 
NZW/BXSB F1, and NZM2410 mice significantly reduced the amount of target-organ damage, without preventing the emergence or deposition of pathogenic autoantibodies. ${ }^{11-13}$ Similarly, in autoimmune-resistant MHCdeficient C57BL/6 (B6) mice, BLyS overexpression led to humoral autoimmunity; however, renal immunopathology and clinical disease did not develop. ${ }^{14}$ These observations suggest that target-organ damage in SLE depends not only on tissue deposition of immune globulins and complement, but also on the inflammatory burden imposed by activated cells and their inflammatory mediators. ${ }^{7}$ Only in the presence of a certain inflammatory microenvironment or an autoimmunity-susceptible genetic background would a BLySdriven immunopathology progress to clinical disease. ${ }^{13}$

In accordance with observations in mice, administration of belimumab for 26 weeks in cynomolgus monkeys yielded a $50 \%-60 \%$ B-cell reduction, most significant in the mature B-cell subset, accompanied by decrease in size of the splenic lymphoid follicles. Reductions were observed at 4-8 weeks and were persistent after cessation of treatment, with return to baseline at 52 weeks. ${ }^{15}$

Among 185 patients with various systemic autoimmune diseases, 95 of whom had SLE, 20\% had significantly elevated plasma BLyS levels compared to healthy controls, and levels of BLyS correlated with IgG levels and anti-dsDNA titers. ${ }^{16}$ Elevated serum BLyS and blood BLyS messenger ribonucleic acid (mRNA) levels were demonstrated in $50 \%-61 \%$ of longitudinally followed SLE patients. ${ }^{17}$ Serum BLyS levels correlated with anti-dsDNA titers in patients with detectable anti-dsDNA in the same study. In addition, BLyS concentrations seem to be related to disease activity in patients with SLE, as measured by SELENA-SLEDAI (SS). ${ }^{18}$ BLyS mRNA levels have been proposed as a better marker of disease activity than serum protein levels. ${ }^{19}$

\section{Clinical trials of belimumab in SLE: phases I and II}

A phase I dose-ranging RCT in 70 SLE patients with stable disease activity was the first to demonstrate belimumab's in vivo safety and provide insight into its pharmacokinetic properties and biological activity. ${ }^{20}$ Belimumab's long elimination half-life, slow clearance, and small volume of distribution are consistent with a fully human monoclonal antibody. ${ }^{20}$

A phase II double-blind RCT subsequently examined the safety, tolerability, biological activity, and efficacy of the drug in 449 SLE patients and a SS score $\geq 4 .{ }^{21}$ Subjects were assigned to either belimumab $(1,4$, and $10 \mathrm{mg} / \mathrm{kg})$ or placebo, administered intravenously on days 0,14 , and 28 and every 28 days for 52 weeks. All subjects also received standard of care treatment, with a stable regimen of steroids, antimalarials, or immunosuppressants for 60 days prior to the first belimumab dose. No significant differences between treatment and placebo groups were attained for either of the coprimary endpoints (\% change in the SS score at 24 weeks and the time to first SLE flare), and no dose response was observed. The time to first flare at weeks $24-52$ was however prolonged with belimumab (154 days in the combined belimumab group compared to 104 days in placebo, $P=0.036$ ), suggesting that the drug can stabilize disease, but requires some time to do so. Significant decrease in mean physician's global assessment (PGA) score at 52 weeks was noted (31\% with belimumab compared to $14 \%$ in placebo, $P=0.0019)$.

Post-hoc analysis of the subgroup of patients with serologically active disease (anti-nuclear autoantibodies [ANA] $\geq 1: 80 \mathrm{and} /$ or anti-dsDNA $\geq 30 \mathrm{IU} / \mathrm{mL}$ ) yielded significantly better responses at 52 weeks in belimumab- versus placebo-treated patients in SS score, PGA, and short form 36 (SF-36) physical component score (PCS). ${ }^{21}$ This patient subgroup had significantly more historic SLE criteria (renal, hematologic, and immunologic) and higher disease activity at baseline compared to ANA-negative patients. ${ }^{22}$ More patients in the ANA-positive group had detectable BLyS levels, whereas this group also had lower serum complement $(P<0.0001)$, higher immunoglobulin levels $(P \leq 0.001)$, and increased number of CD19+/27+/38++ plasma cells $(P=0.1)$, all being suggestive of disease activity due to B-cell dysfunction. ${ }^{22}$

Critical analysis of the phase II belimumab trial data led to creation of the SLE responder index, a novel robust tool that could better define clinical meaningful changes in disease activity and be used as the primary endpoint in SLE trials. ${ }^{23}$ In this compound index, SS scores were utilized to define global improvement, British Isles Lupus Assessment Group (BILAG) domain scores to ensure no significant worsening in previously unaffected systems, and PGA to ensure that improvement in disease activity was not achieved to the detriment of the patient's overall condition. The systemic response index (SRI) was defined by: $\geq 4$-point reduction from baseline in SS score, no new A or $\geq 2$ B BILAG organ domain scores, and no $\geq 0$.3-point deterioration from baseline in PGA. BILAG scores A and B, respectively, indicate a severe and moderate flare in any of the eight organ domains of the index, which was designed based on the physician's intention to treat. ${ }^{24}$ By retrospective application of the SRI in the seropositive patients of the 
phase II belimumab trial, treatment with belimumab yielded a $46 \%$ response at week 52 , compared to $29 \%$ in the placebo group $(P=0.006)$. SRI responses remained independent of baseline autoantibody subtype. ${ }^{23}$ This index fulfilled the FDA-suggested criteria for clinical endpoints in RCT in $\mathrm{SLE}^{25}$ and was approved by regulatory authorities as the primary endpoint for two large phase III studies of belimumab, belimumab international SLE study (BLISS)-52 and BLISS-76, with the potential to be used again in similarly designed clinical trials.

\section{Clinical trials of belimumab in SLE: phase III}

Promising results of the phase II belimumab trial encouraged two larger global phase III trials of belimumab versus placebo, which recruited seropositive (ANA $\geq 1: 80$ or anti-dsDNA $\geq 30 \mathrm{IU} / \mathrm{mL}$ at study entry) SLE patients with stable disease receiving standard of care treatment. ${ }^{26}$ Patients had a SS score $\geq 6$ and were stable on standard of care therapy for $\geq 30$ days upon study entry. In both studies, patients were randomized to belimumab ( 1 or $10 \mathrm{mg} / \mathrm{kg}$ ) or placebo on days 0,14 , and 28 and every 28 days for 48 weeks. BLISS-52 (NCT00424476) ${ }^{27}$ was conducted between May 2007 and July 2009 and included 865 SLE patients enrolled in North America and Western and Central Europe. BLISS-76 (NCT00410384) ${ }^{28}$ was conducted between February 2007 and February 2010 enrolling 819 patients in Central and Eastern Europe, Latin America, and Asia Pacific. Disease activity was assessed by SS, BILAG, and the SS Flare Index (SFI). SS and BILAG domain scores were measured every 4 weeks. ${ }^{29}$ In accordance with the EULAR guidelines, health-related quality of life was assessed by use of the SF-36 domain, Physical and Mental Component Summary (PCS and MCS) scores, and the FACIT-Fatigue questionnaire. ${ }^{30}$ The primary endpoint in both trials was the SRI at 52 weeks. Secondary endpoints included the percentage of subjects with a 4-point reduction from baseline in SS score at 52 weeks, the mean change in PGA at 24 weeks, the mean change in SF-36 PCS at 24 weeks, and the percentage of subjects taking $>7.5 \mathrm{mg} / \mathrm{d}$ of prednisone at baseline whose average prednisone dose was reduced by $\geq 25 \%$ or to $\leq 7.5 \mathrm{mg}$ /day during weeks 40-52. The SRI at 76 weeks was an additional secondary endpoint in BLISS-76. Intention to treat principles was followed in data analysis.

Mean values of baseline disease characteristics were similar across the belimumab and placebo groups within each study, though variations were observed among the BLISS-52 and BLISS-76 cohorts (Table 1). ${ }^{31-33}$ Disease duration was longer
Table I Mean values of baseline disease characteristics of SLE patients in the BLISS-52 and BLISS-76 trials

\begin{tabular}{llll}
\hline $\begin{array}{l}\text { Mean baseline } \\
\text { characteristics }\end{array}$ & BLISS-52 & BLISS-76 & $\begin{array}{l}\text { Combined } \\
\text { cohort }\end{array}$ \\
\hline Disease duration, years & 5.3 & 7.5 & 6.4 \\
SELENA-SLEDAI & 9.8 & 9.7 & 9.7 \\
BILAG IA/2B, \% & 58 & 64 & 61 \\
ANA positive, \% & 95 & 92 & \\
Anti-dsDNA positive, \% & 75 & 64 & 69.4 \\
Proteinuria & 25 & 16 & 20.1 \\
(>0.5 gr/24 h), \% & & & \\
Low C4, \% & 59 & 53 & 56.1 \\
Antimalarial use, \% & 67 & 63 & 65.3 \\
Prednisone equivalent & 69 & 46 & 58.0 \\
dose $\geq 7.5$ mg/day, \% & & & \\
Immunosuppressant & 42 & 56 & 48.7 \\
use, \% & & & \\
\hline
\end{tabular}

Note: Values were similar across treatment groups for each trial.

Abbreviations: SLE, systemic lupus erythematosus; BLISS, belimumab international SLE study.

in BLISS-76 (7.5 vs 5.3 years in BLISS-52). Serologically, active lupus by means of anti-dsDNA antibody positivity was more frequent in BLISS-52, but more details on patient's baseline immunologic characteristics have not yet been published. SS and BILAG organ system involvement at baseline were generally similar in the belimumab and placebo groups and between studies. ${ }^{29}$ The most common SS domains involved were dermal (82\%), immunologic (80\%), musculoskeletal (65\%), and renal (16\%). The most common BILAG domains involved were musculoskeletal $(60 \%)$, mucocutaneous $(59 \%)$, hematology $(16 \%)$, renal $(11 \%)$, and general (11\%). ${ }^{29}$ Use of background medications differed at baseline, with higher steroid and lower immunosuppressant use in BLISS-52 indicating different approaches to therapy among different institutions and treatment locations. Disclosures on the BLISS trials design and results would be helpful, as data have been presented in abstract form and through the manufacturer's website to date, without getting subject to the rigors of peer-reviewed publications.

A significantly higher rate of SRI at 52 weeks was noted for both belimumab groups compared to placebo in BLISS-52. This was reached at 24-28 weeks and was sustained throughout the study (Table 2). ${ }^{26,31}$ Combined analysis of the two BLISS trials yielded significant differences in SRI rates at week 52 for both belimumab groups compared to placebo (Table 3). ${ }^{33}$ Effects in BLISS-76 were, however, only modest, with significantly higher SRI rates at 52 weeks reached only in the $10 \mathrm{mg} / \mathrm{kg}$ belimumab group $(P=0.02$ compared to placebo) and not sustained at 76 weeks (Table 4). ${ }^{26,32,34}$ Post hoc exploratory analyses using $>4$-point 
Table 2 Belimumab effects on primary and secondary endpoints in BLISS-52

\begin{tabular}{|c|c|c|c|c|c|}
\hline BLISS 52: endpoints & $\begin{array}{l}\text { SOC }+ \text { placebo } \\
(\mathbf{N}=\mathbf{2 8 7})\end{array}$ & $\begin{array}{l}\text { SOC + belimumab } \\
\text { I mg/kg }(\mathrm{N}=\mathbf{2 8 8})\end{array}$ & $\mathbf{P}$ & $\begin{array}{l}\text { SOC + belimumab } \\
10 \mathrm{mg} / \mathrm{kg}(\mathrm{N}=290)\end{array}$ & $\boldsymbol{P}$ \\
\hline SRI at week $52, \mathrm{n}(\%)^{*}$ & $125(44)$ & I48 (5I) & 0.013 & $167(58)$ & 0.0006 \\
\hline - SS $\geq 4$-point reduction (\%)** & $132(46)$ & $153(53)$ & 0.019 & $169(58)$ & 0.0024 \\
\hline - $\leq 0.3$-point PGA worsening (\%) & $199(69)$ & 227 (79) & 0.0078 & $231(80)$ & 0.0048 \\
\hline - No new IA/2B BILAG (\%) & $210(73)$ & $227(79)$ & 0.086 & $236(8 I)$ & 0.018 \\
\hline Mean \% PGA improvement at week $24 * *$ & 22 & 30 & 0.034 & 37 & 0.0001 \\
\hline $\begin{array}{l}\text { SFI flare, \% }(\mathrm{HR}) / \text { median time to } \\
\text { first flare, days }\end{array}$ & $80 / 84$ & $7 I(0.75) / I 26$ & 0.0026 & $71(0.76) / 119$ & 0.0036 \\
\hline Severe SFI flare at 52 weeks, \% (HR) ${ }^{* *}$ & 23 & $18(0.76)$ & 0.13 & $14(0.57)$ & 0.0055 \\
\hline New IA/2B BILAG, \% (HR) & 30 & $27(0.89)$ & 0.48 & $19(0.58)$ & 0.0016 \\
\hline $\begin{array}{l}\text { Prednisone dose reduction from }>7.5 \mathrm{mg} / \text { day } \\
\text { at baseline, by } 25 \% \text { or to } \leq 7.5 \mathrm{mg} / \text { day, } \\
\text { during weeks } 40-52, \mathrm{n}(\%)^{* *}\end{array}$ & $23(12)$ & $42(2 \mathrm{I})$ & 0.025 & $38(19)$ & 0.053 \\
\hline $\begin{array}{l}\text { Prednisone dose increase from } \leq 7.5 \mathrm{mg} / \text { day } \\
\text { to }>7.5 \mathrm{mg} / \text { day at week } 52, \mathrm{n}(\%)\end{array}$ & $34(36)$ & $25(30)$ & 0.56 & $17(20)$ & 0.020 \\
\hline $\begin{array}{l}\text { SF-36 PCS change from baseline } \\
\text { at week } 52 \text {, mean (SD) }\end{array}$ & $+3.0(0.5)$ & $+4.2(0.5)$ & $<0.05$ & $+4.2(0.5)$ & $<0.05$ \\
\hline $\begin{array}{l}\text { FACIT-fatigue score change from } \\
\text { baseline at week } 52 \text {, mean (SD) }\end{array}$ & $+2.1(0.6)$ & $+3.9(0.6)$ & $<0.001$ & $+4.8(0.6)$ & $<0.001$ \\
\hline
\end{tabular}

Notes: *Primary endpoint; **Prespecified secondary endpoints.

Abbreviations: BLISS, belimumab international SLE study; SRI, systemic response index; SS, SELENA-SLEDAI; PGA, physician's global assessment; HR, hazard ratio; SD, standard deviation; SFI, SS flare index; SF-36, short form 36; PCS, physical component score; SOC, standard of care.

SS reductions showed a greater treatment effect on SRI rates for belimumab compared to placebo in both BLISS cohorts. ${ }^{26,34}$ Whether raising the SS threshold for improvement in disease activity would be truly meaningful remains to be addressed in future trials. Furthermore, the less favorable outcomes in BLISS-76 could be congruent with the notion that belimumab is less effective in mice and perhaps humans with late, established disease. ${ }^{7}$ Indeed, decreased efficacy of belimumab, despite B-cell depletion, was observed in lupusprone mice once autoreactive $\mathrm{T}$ cells and dendritic cells had

Table 3 Combined efficacy results of BLISS-52 and BLISS-76

\begin{tabular}{|c|c|c|c|c|c|}
\hline Endpoints & $\begin{array}{l}\text { SOC + placebo } \\
(N=562)\end{array}$ & $\begin{array}{l}\text { SOC + belimumab } \\
\text { I mg/kg }(N=559)\end{array}$ & $\mathbf{P}$ & $\begin{array}{l}\text { SOC + belimumab } \\
10 \mathrm{mg} / \mathrm{kg}(\mathrm{N}=563)\end{array}$ & $\boldsymbol{P}$ \\
\hline SRI at week 52, n (\%) & $218(38.8)$ & $258(46.2)$ & $<0.0$ I & $285(50.6)$ & $<0.0001$ \\
\hline - SS $\geq 4$-point reduction (\%) & $230(40.9)$ & $269(48.1)$ & $<0.01$ & $297(52.8)$ & $<0.05$ \\
\hline$\bullet \leq 0.3$-point PGA worsening (\%) & $372(66.2)$ & $424(75.8)$ & $<0.001$ & $420(74.6)$ & $<0.01$ \\
\hline - No new IA/2B BILAG (\%) & $389(69.2)$ & $429(76.7)$ & $<0.01$ & $425(75.5)$ & $<0.05$ \\
\hline Mean \% PGA improvement at week 24 & 24.3 & 28.8 & ns & 32.3 & $<0.01$ \\
\hline Mean \% PGA improvement at week 52 & 27.1 & 36.7 & $<0.01$ & 37.8 & $<0.001$ \\
\hline $\begin{array}{l}\text { SFI flare, \% (HR)/median time to } \\
\text { first flare, days }\end{array}$ & $81.5 / 84$ & $74.6(0.82) / 110$ & $<0.01$ & $74.6(0.84) / 110$ & $<0.05$ \\
\hline Severe SFI flare, \% (HR) & 23.7 & I7.0 (0.7I) & $<0.05$ & $15.6(0.64)$ & $<0.01$ \\
\hline New IA/2B BILAG, \% (HR) & 32.0 & $27.2(0.83)$ & ns & $24.9(0.75)$ & $<0.05$ \\
\hline New IA BILAG, \% (HR) & 23.1 & $19.0(0.81)$ & ns & $16.2(0.67)$ & $<0.01$ \\
\hline $\begin{array}{l}\text { Prednisone dose reduction from } \\
>7.5 \mathrm{mg} / \text { day at baseline, by } 25 \% \text { or to } \\
\leq 7.5 \mathrm{mg} / \text { day, during weeks } 40-52 \text {, n (\%) }\end{array}$ & $39(12.3)$ & $67(20.1)$ & $<0.01$ & $58(17.9)$ & $<0.05$ \\
\hline $\begin{array}{l}\text { Prednisone dose increase from } \\
\leq 7.5 \text { to }>7.5 \mathrm{mg} / \text { day at week } 52, \mathrm{n}(\%)\end{array}$ & $82(33.6)$ & $58(25.8)$ & ns & $62(25.9)$ & ns \\
\hline $\begin{array}{l}\text { SF-36 PCS change from baseline at } \\
\text { week } 52 \text {, mean (SD) }\end{array}$ & $+2.9(0.3)$ & $+4.3(0.4)$ & $<0.01$ & $+3.8(0.3)$ & $<0.05$ \\
\hline $\begin{array}{l}\text { FACIT-fatigue score change from } \\
\text { baseline at week } 52 \text {, mean (SD) }\end{array}$ & $+2.5(0.4)$ & $+4.8(0.4)$ & $<0.001$ & $+4.7(0.4)$ & $<0.001$ \\
\hline
\end{tabular}

Abbreviations: BLISS, belimumab international SLE study; SRI, systemic response index; SS, SELENA-SLEDAI; PGA, physician's global assessment; HR, hazard ratio; SD, standard deviation; SFI, SS flare index; SF-36, short form 36; PCS, physical component score; SOC, standard of care. 
Table 4 Belimumab's effects on primary and secondary endpoints in BLISS-76

\begin{tabular}{|c|c|c|c|c|c|}
\hline BLISS 76: endpoints & $\begin{array}{l}\text { SOC + placebo } \\
(\mathbf{N}=275)\end{array}$ & $\begin{array}{l}\text { SOC + belimumab } \\
\text { I } \mathrm{mg} / \mathrm{kg}(\mathrm{N}=27 \mathrm{I})\end{array}$ & $\boldsymbol{P}$ & $\begin{array}{l}\text { SOC + belimumab } \\
10 \mathrm{mg} / \mathrm{kg}(\mathrm{N}=273)\end{array}$ & $P$ \\
\hline SRI at week 52, n (\%)* & $93(34)$ & IIO (4I) & 0.104 & II 8 (43) & 0.021 \\
\hline - SS $\geq 4$-point reduction (\%)** & $98(36)$ & $116(43)$ & 0.087 & $128(47)$ & 0.006 \\
\hline - $\leq 0.3$-point PGA worsening (\%) & $173(63)$ & $197(73)$ & 0.012 & $189(69)$ & 0.126 \\
\hline - No new IA/2B BILAG (\%) & $179(65)$ & $203(75)$ & 0.011 & $189(69)$ & 0.319 \\
\hline SRI at week $76, \mathrm{n}(\%)^{* *}$ & $89(32.4)$ & $106(39.1)$ & 0.13 & $105(38.5)$ & 0.11 \\
\hline$S S \geq 4$-point reduction at week $76(\%)$ & $93(33.8)$ & II $4(42.1)$ & ns & $113(4 \mid .4)$ & ns \\
\hline Mean $\%$ PGA improvement at week $24 * *$ & 26.2 & 28.1 & 0.514 & 27.6 & 0.468 \\
\hline SFI flare, \% (HR)/median time to first flare, days & $83 / 82$ & $79(0.89) / 85$ & 0.232 & $79(0.93) / 84$ & 0.479 \\
\hline Severe SFI flare, \% (HR) & 24 & $16(0.64)$ & 0.023 & $18(0.72)$ & 0.086 \\
\hline SFI flare at weeks $24-76, \%$ (HR) & 81.2 & $75.9(0.84)$ & ns & $73.3(0.78)$ & $<0.05$ \\
\hline Severe SFI flare at weeks $24-76, \%(H R)$ & 21.8 & $12.7(0.55)$ & $<0.01$ & I5.7 (0.70) & ns \\
\hline New BILAG IA/2B flare, \% (HR) & 34 & $28(0.78)$ & 0.119 & $32(0.93)$ & 0.613 \\
\hline $\begin{array}{l}\text { Prednisone dose reduction from }>7.5 \mathrm{mg} / \text { day } \\
\text { at baseline, by } 25 \% \text { or to } \leq 7.5 \mathrm{mg} / \text { day, } \\
\text { during weeks } 40-52, \mathrm{n}(\%)\end{array}$ & $16(13)$ & $25(19)$ & 0.203 & $20(17)$ & 0.532 \\
\hline $\begin{array}{l}\text { Prednisone dose reduction from }>7.5 \mathrm{mg} / \text { day } \\
\text { at baseline, by } 25 \% \text { or to } \leq 7.5 \mathrm{mg} / \text { day, } \\
\text { during weeks } 64-76, \mathrm{n}(\%)\end{array}$ & $22(17.5)$ & $35(26.9)$ & ns & $29(24.2)$ & ns \\
\hline $\begin{array}{l}\text { Prednisone increase from } \leq 7.5 \text { to }>7.5 \mathrm{mg} / \text { day } \\
\text { at week } 52, \mathrm{n}(\%)\end{array}$ & $48(32)$ & $33(23)$ & 0.109 & $45(29)$ & 0.608 \\
\hline $\begin{array}{l}\text { Prednisone increase from } \leq 7.5 \text { to }>7.5 \mathrm{mg} / \text { day } \\
\text { at week } 76, \mathrm{n}(\%)\end{array}$ & $27(18.1)$ & $19(13.5)$ & ns & $18(11.8)$ & ns \\
\hline $\begin{array}{l}\text { SF- } 36 \text { PCS change from baseline } \\
\text { at week } 52 \text {, mean (SD) }\end{array}$ & $+2.85(0.5)$ & $+4.37(0.5)$ & 0.012 & $+3.41(0.47)$ & 0.51 \\
\hline $\begin{array}{l}\text { FACIT-fatigue score change from baseline } \\
\text { at week } 52 \text {, mean (SD) }\end{array}$ & $+2.86(0.66)$ & $+5.76(0.66)$ & 0.002 & $+4.63(0.63)$ & 0.138 \\
\hline $\begin{array}{l}\text { FACIT-fatigue score change from baseline } \\
\text { at week } 76 \text {, mean }\end{array}$ & +3.16 & +5.23 & $<0.05$ & +5.0 & ns \\
\hline
\end{tabular}

Notes: *Primary endpoint; **Prespecified secondary endpoints.

Abbreviations: BLISS, belimumab international SLE study; SRI, systemic response index; SS, SELENA-SLEDAl; PGA, physician's global assessment; HR, hazard ratio; SD, standard deviation; SFI, SS flare index; SF-36, short form 36; PCS, physical component score; SOC, standard of care.

formed and multiple active amplification pathways had been established. ${ }^{7}$

Several secondary endpoints of disease activity and flares were examined, with varied results in the two trials. Consistent with achievement of significant SRI rates in BLISS-52 were changes in disease activity indices, including SS and PGA. The percentage of patients achieving a 4-point reduction from baseline in SS score at week 52, the mean percentage change from baseline in PGA at week 24, and the percentage of patients having $\leq 0.3$ PGA worsening from baseline at week 52 were all significantly higher for both belimumab groups compared to placebo (Table 2). ${ }^{31}$ Similarly, in combined analysis of the two BLISS trials, changes in the individual components of the SRI at 52 weeks were statistically significant for both belimumab groups compared to placebo (Table 3) ${ }^{33}$ Additionally, mean changes in PGA were statistically significant compared to placebo for the combined $10 \mathrm{mg} / \mathrm{kg}$ group at 24 weeks and for both belimumab groups in 52 weeks (Table 3). ${ }^{33}$ Effects on secondary endpoints were, however, more limited in BLISS-76 (Table 4). A trend in the percentage of patients with a 4-point reduction in SS at week 52 was observed in the $1 \mathrm{mg} / \mathrm{kg}$ group $(P=0.087)$, reaching statistical significance over placebo in the $10 \mathrm{mg} / \mathrm{kg}$ group $(P=0.006) .{ }^{32}$ The percentage of patients having $\leq 0.3$ PGA worsening at week 52 was statistically increased over placebo only for the $1 \mathrm{mg} / \mathrm{kg}$ group $(P=0.012)$. No difference in the percentage improvement in PGA at week 24 was observed in the treatment arms compared to placebo (Table 4). ${ }^{32}$

Considering that some SLE manifestations, like thrombocytopenia, may be more susceptible to belimumab, the distribution of SS and BILAG domain scores in the BLISS trials is of great interest. ${ }^{12}$ Changes in particular organ domains were recently published for the combined BLISS cohort. Significant improvement from baseline at week 52 was noted in the central nervous system, vascular, musculoskeletal, immunologic, and dermal domains of SS with belimumab compared to placebo. ${ }^{29}$ Among patients 
without involvement in a specific organ system at baseline, there was significantly less worsening at week 52 in the immunologic, renal, and hematologic domains. A significant improvement from baseline at week 52 was noted in the musculoskeletal and mucocutaneous domains and a trend $(P<0.07)$ in the vasculitis BILAG domain with belimumab compared to placebo. There was also significantly less worsening in the hematology BILAG domain and a favorable trend in the vasculitis and renal domains. ${ }^{29}$

Significant improvements were seen in both belimumab groups of BLISS-52 in the SFI flare rate and time to first flare, although the severe SFI flare rate and the rate of new BILAG $1 \mathrm{~A} / 2 \mathrm{~B}$ scores at 52 weeks were significantly reduced only in the $10 \mathrm{mg} / \mathrm{kg}$ belimumab group (Table 2). ${ }^{31}$ In the combined BLISS trials data analysis, the SFI flare rate, the severe SFI flare rate, and the time to first flare were statistically improved for both belimumab groups, whereas new BILAG $1 \mathrm{~A} / 2 \mathrm{~B}$ and new BILAG $1 \mathrm{~A}$ score rates were reduced only in the $10 \mathrm{mg} / \mathrm{kg}$ belimumab group compared to placebo (Table 3 ). ${ }^{33}$ In BLISS-76, the SFI flare rate and the time to first flare did not, however, differ in either belimumab group, whereas the severe SFI flare rate was reduced in the $1 \mathrm{mg} / \mathrm{kg}$ belimumab group compared to placebo. ${ }^{32}$ Of note, the SFI flare rate at weeks 24-76 was significantly improved in the $10 \mathrm{mg} / \mathrm{kg}$ belimumab group, whereas the severe SFI flare rate over weeks 24-76 significantly improved in the $1 \mathrm{mg} / \mathrm{kg}$ group compared to placebo (Table 4). ${ }^{34}$ No difference in the rate of new BILAG 1A/2B scores was noted in BLISS-76 (Table 4). ${ }^{32,34}$ Secondary outcomes should, nonetheless, be interpreted with caution, bearing in mind the usually inadequate statistical power for their determination. The inverse dose-response relationship for some of the observed results further underlines the effects of random variation and multiple comparisons in studies with multiple endpoints. ${ }^{35}$ In the absence of a reasonable, biologically plausible explanation, such differences may well be the result of chance.

In BLISS-52, a steroid-sparing effect of the belimumab was observed among patients on $>7.5 \mathrm{mg}$ of daily prednisone at baseline, who had their average dose reduced by $25 \%$ or to $\leq 7.5 \mathrm{mg}$ /day during weeks 40-52 (Table 2). ${ }^{31}$ Among patients on $\leq 7.5 \mathrm{mg}$ /day at baseline, significantly fewer patients increased their prednisone dose to $>7.5 \mathrm{mg} /$ day at week 52 in the $10 \mathrm{mg} / \mathrm{kg}$ belimumab group $(P=0.02$ compared to placebo) (Table 2). ${ }^{31}$ In combined BLISS trials analysis, the same steroid-sparing effect was observed during weeks 40-52 for subjects on $>7.5 \mathrm{mg} /$ day of prednisone at baseline ( $P<0.05$ for both belimumab groups compared to placebo) (Table 3). ${ }^{32,33}$ There was no difference between groups, in the percentage of patients on $<7.5 \mathrm{mg}$ daily at baseline that increased their steroid dosage at week 52. ${ }^{33}$ Nonsignificant differences in these endpoints were, however, observed among belimumab and placebo in BLISS-76 (Table 4). ${ }^{32,34}$

Patient-reported outcomes pertinent to HRQoL were complementary to physician-centered indices in the BLISS trials. ${ }^{36,37}$ Mean improvement from baseline in SF-36 PCS at week 24, a major prespecified endpoint, did not differ between treatment arms and placebo in any of the BLISS trials. ${ }^{38}$ In BLISS-52, differences in PCS, physical functioning, and bodily pain were significant at 52 weeks for both belimumab groups compared to placebo (Table 2). ${ }^{30}$ Both belimumab groups in this study demonstrated significant improvement in FACIT-Fatigue scores by week 52 compared to placebo (Table 2), with an early sustained response observed in the $10 \mathrm{mg} / \mathrm{kg}$ belimumab group $(P=0.0015$ at 8 weeks, $P=0.024$ at 36 weeks, and $P=0.0003$ at 52 weeks compared to placebo). ${ }^{30}$ Similar significant improvements in SF-36 PCS at 52 weeks and in FACIT-Fatigue at 52 and 76 weeks were observed in the $1 \mathrm{mg} / \mathrm{kg}$ belimumab group of BLISS-76 (Table 4) ${ }^{32,34}$ On the pooled BLISS data analysis, the mean change from baseline in SF-36 PCS and FACIT-Fatigue scores at 52 weeks were statistically significant for both belimumab groups compared to placebo (Table 3 ). ${ }^{38}$

Despite the not always substantial improvements in clinical outcomes, changes in immunologic parameters were impressive in the BLISS trials. ${ }^{39}$ Significant reductions in immunoglobulins (immunoglobulin $\mathrm{G}$ [ $\mathrm{IgG}]$, immunoglobulin $\mathrm{M}$ (IgM), immunoglobulin A [IgA]) ( $P<0.0001$ for both belimumab groups $)$ and autoantibodies (anti-dsDNA IgG, anti-Sm IgG, anti-ribosomal P IgG $[P=0.0001,<0.005,0.02$, respectively, for the $1 \mathrm{mg} / \mathrm{kg}$ group and $<0.0001$ for the $10 \mathrm{mg} / \mathrm{kg}$ group], anti-cardiolipin $\operatorname{IgA}[P=0.01$ for the $10 \mathrm{mg} / \mathrm{kg}$ group $]$ ) and normalization in hyperglobulinemia $(>1618 \mathrm{~g} / \mathrm{L}$ ) (in $50 \%$ of the $1 \mathrm{mg} / \mathrm{kg}$ group and $48.9 \%$ of the $10 \mathrm{mg} / \mathrm{kg}$ group versus $19.1 \%$ in placebo $[P<0.0001])$ were noted at 52 weeks for both belimumab groups of BLISS-52 compared to placebo. ${ }^{39}$ Similar were the effects on seroconversion rates at week 52 for patients seropositive for anti-dsDNA, anti-Sm, antiribosomal $\mathrm{P}$, and/or anti-cardiolopin $(\mathrm{IgG}+\operatorname{IgM})$ antibodies at baseline $(P=0.01$ for the $10 \mathrm{mg} / \mathrm{kg}$ group; results in the $1 \mathrm{mg} / \mathrm{kg}$ group reached significance only for anti-dsDNA, anti-ribosomal $\mathrm{P}$, and anti-cardiolipin IgG antibodies). ${ }^{39}$ In the pooled analysis of BLISS trials, similar results were observed. ${ }^{40}$ Median percentage reductions in anti-dsDNA, anti-Sm, anti-cardiolipin IgG, and anti-P antibody serum levels and seroconversion rates from positive to negative 
were statistically significant for both belimumab doses compared to placebo. ${ }^{40}$ Significant median percent reductions of baseline anti-dsDNA levels at 76 weeks were also observed in both belimumab groups of BLISS-76 $\left(P<0.0001\right.$ compared to placebo).${ }^{34}$ Seroconversion from positive to negative anti-dsDNA was significant only in the $1 \mathrm{mg} / \mathrm{kg}$ belimumab group. ${ }^{34}$ Given the correlations of anti-dsDNA titers with BLyS levels and SLE disease activity, post-hoc exploratory analysis of clinical responses in this patient subgroup would be of interest.

Consistent with the effects of belimumab on antibody production were changes in complement components known to have a major role in antibody-mediated tissue damage and SLE disease activity. Significantly greater increases in $\mathrm{C} 3$ and $\mathrm{C} 4$ levels and a higher \% normalization of low $\mathrm{C} 3$ and $\mathrm{C} 4$ were noted in belimumab-treated patients compared to placebo-treated patients at 52 weeks in BLISS-52 $(P<0.005$ for both belimumab groups, except nonsignificant \% normalization of C3 with belimumab $1 \mathrm{mg} / \mathrm{kg}$ compared to placebo). ${ }^{39}$ Similar increases and normalization in $\mathrm{C} 4$ titers were observed with belimumab compared to placebo in BLISS-76 $(P<0.0001$ and $P<0.001$ for belimumab 10 and $1 \mathrm{mg} / \mathrm{kg}$, respectively, compared to placebo), whereas significant changes and normalization in $\mathrm{C} 3$ titers were noted only in the $10 \mathrm{mg} / \mathrm{kg}$ group. ${ }^{34} \mathrm{In}$ pooled data analysis, belimumab-treated patients had significantly greater increases in C3 and C4 levels $(P<0.05$ compared to placebo), and a dose-response relation was observed. ${ }^{40}$

In accordance with results of animal studies and the phase II belimumab trial, significant reductions in CD20+ B cells (including CD20+/CD27- naive cells, CD20+/CD69+ activated cells, and CD20+/CD138+ plasmacytoid cells) were observed with belimumab over placebo in BLISS-76. ${ }^{34}$ CD20-/CD138+ plasma cells, CD20-/CD27 ${ }^{\text {hi }}$ short-lived plasma cells, and CD19+/CD27 $/$ hi $/$ 38 ${ }^{\text {hi }}$ cell subsets showed similar reductions from baseline, significant only in the $10 \mathrm{mg} / \mathrm{kg}$ group compared to placebo. On the contrary, increases in CD20+/CD27+ memory cells were noted, reaching statistical significance in the $10 \mathrm{mg} / \mathrm{kg}$ group compared to placebo. ${ }^{34}$ Regarding effects on T cells, significant increases in $\mathrm{CD} 3+/ 4+, \mathrm{CD} 3+/ 8+$, and total CD3+ subsets at 76 weeks were observed with belimumab compared to placebo. ${ }^{34}$ Significant reductions of various circulating B-cell subsets, but not T-cell subsets, were similarly observed in the combined analysis of the belimumab trials. ${ }^{40}$ Memory B cells increased $100 \%$ by week 8 and gradually decreased toward baseline levels through week 52. A modest expansion of T-cell subsets was observed at week 52, but this is considered to be a secondary effect of the B-cell reductions. ${ }^{40}$

Changes in immunologic parameters, although concordant, did not translate to such impressive changes in disease activity, highlighting the biological complexity of SLE. Multiple pathways are contributory to histologic damage and clinical disease in SLE, not all of which are sensitive to BLyS. Inflammation accelerated by INFa was resistant to BLyS blockade in animal models. ${ }^{7}$ Interactions of B cells with $\mathrm{T}$ cells and other cell types significantly influence response to BLyS blockade as suggested by the enhanced efficacy of combined B- and T-cell inhibition in murine lupus. ${ }^{11}$ Autoantibody production by long-lived plasma cells is independent of BLyS and thus resistant to BLyS blockade. ${ }^{7,12}$ Accordingly, the clinical effects of therapeutic BLyS neutralization may vary widely across the heterogeneous population of SLE.

Compounding its clinical efficacy, belimumab demonstrated a favorable safety profile in the BLISS trials, an argument favoring its use over other commonly administered immunosuppressive drugs in SLE. ${ }^{2}$ In both BLISS-52 and BLISS-76, the overall rates of adverse events, deaths, serious adverse events, infections, and laboratory abnormalities were comparable among belimumab and placebo. ${ }^{26,31,32,34}$ A slightly higher risk of hypersensitivity infusion reactions was reported in BLISS-52 (1.6\% with belimumab compared to $0.3 \%$ with placebo). ${ }^{26,31}$ Comparably, serious and severe infusion reactions were modestly increased with belimumab compared to placebo in BLISS-76 $(1.1 \%$ in the $10 \mathrm{mg} / \mathrm{kg}$ group vs $0.4 \%$ in placebo). ${ }^{32,34}$ No malignancies were reported in BLISS-52, whereas six occurred in BLISS-76 (one on placebo, four on $1 \mathrm{mg} / \mathrm{kg}$, two on $10 \mathrm{mg} / \mathrm{kg}$ ). ${ }^{26,32,34}$ Further insight into belimumab's safety is expected by extension of the BLISS trials.

Belimumab's effects on antibody production to pneumococcal, tetanus, and influenza vaccines, administered prior to study entry, were examined in BLISS-76. ${ }^{41}$ Patients receiving a pneumococcal $(n=73)$ or tetanus $(n=81)$ vaccine within 5 years or an influenza vaccine $(n=146)$ within 1 year prior to study entry were tested for vaccine antibody levels (IgG) at baseline and week 52. Endpoints included the percentage change in antibody levels from baseline to week 52 in patients with preexisting antibodies and the proportion of patients with protective antibodies at baseline who maintained protective levels at week 52 . Consistent with the preservation of the memory B-cell compartment (CD20+/27+ B cells), belimumab did not cause a statistically significant decrease in preexisting antibodies to 
pneumococcal and tetanus vaccines. Most patients were able to maintain protective titers to these vaccines, and changes were similar across treatment groups. Preexisting antibody responses to seasonal influenza vaccines were generally not affected by belimumab, although a statistical, but not clinically relevant, difference (less than onefold median change in titer) between treatment groups was observed for some antigens. The percentage of patients that maintained protective specific titers at the end of 52 weeks was similar in the belimumab and placebo treatment groups. ${ }^{41}$

\section{Extension studies}

In parallel with the BLISS trials, a 5-year extension, uncontrolled arm of the phase II belimumab study has generated additional data on efficacy and safety over 1415 patient-years of exposure. ${ }^{42,43}$ In an initial 24-week extension phase beginning at week 56 , placebo subjects were administered belimumab $10 \mathrm{mg} / \mathrm{kg}$, while the dose in belimumab-treated patients was maintained or increased to $10 \mathrm{mg} / \mathrm{kg}$. A total of $19 \%$ of patients dropped out during the placebo-controlled portion of the study, whereas 296 were enrolled at week 80 in the open-label, uncontrolled extension phase of belimumab at $10 \mathrm{mg} / \mathrm{kg}$ administered every 28 days. The study discontinuation rate was 3\%-9\% per year. The baseline characteristics of patients remaining in the trial were similar to those that discontinued the drug. Safety was monitored monthly with reporting of adverse events and flare rates in 6-month intervals. The SS, SFI, and SRI were utilized for measurements of disease activity. All efficacy assessments were limited to the seropositive patient subgroup $(\mathrm{n}=321)$, who were similar to the patients enrolled in the phase III trials. SRI was reached in $46 \%$ seropositive belimumab-treated patients at 1 year (vs $29 \%$ in placebo, $P<0.05$ ), and response was sustained in $55 \%-59 \%$ from 1.5 to 5 years. ${ }^{42,43}$ The slow onset, sustained improvement over time was consistent with belimumab's latency in B-cell depletion. The frequency of new $1 \mathrm{~A} / 2 \mathrm{~B}$ BILAG flares decreased from $30 \%$ at 0.5 year to $23 \%$ at 1 year (vs 33\% and 25\%, respectively, with placebo) and to $11 \%$ at 5 years; flares by SFI decreased from $72 \%$ (severe $13 \%$ ) at 0.5 year to $62 \%$ (severe $8 \%$ ) at 1 year (vs $76 \%$ [severe $9 \%$ ] and 73\% [severe 11\%], respectively, with placebo) and to $22 \%$ (severe $1.6 \%$ ) at 5 years. A steroidsparing effect of belimumab $10 \mathrm{mg} / \mathrm{kg}$ on seropositive patients was noted, as the proportion of patients that decreased their prednisone dose to $<7.5 \mathrm{mg}$ /day continued to increase over 3 years, whereas the proportion of patients requiring a steroid dose increase remained low. ${ }^{44}$
The incidence of adverse effects was generally stable or decreased over 5 years. ${ }^{42}$

Longitudinal measurements of antibody and immunoglobulin titers and complement levels over 3 years were compatible with improvements in SLE disease activity and consistent with immunologic changes in the BLISS trials. ${ }^{45}$ Maximal median reductions of immunoglobulin isotypes were reached in the first 8-24 weeks and stabilized or further declined with continued belimumab treatment. Among patients with hypergammaglobulinemia, 47\% normalized on belimumab at week $52(P=0.0062$ compared to placebo) and up to $57 \%$ over 3 years. Complement levels increased in all SLE patients with low levels at baseline who got treated with belimumab. Belimumab-treated patients were more likely, than placebo, to have a significant decrease in anti-dsDNA antibodies ( $\geq 50 \%$ or conversion to negative) ( $30 \%$ vs $17 \%$ at 52 weeks, $P=0.015$ ), a difference that was further increased at week 160 (47\%). The percentage of patients with significant anti-dsDNA reduction was higher in clinical responders (using SRI) than nonresponders $(P<0.05$ at weeks 52 and 128). Seroconversion rates for anti-Sm or anti-RNP antibodies over weeks 52-160 were, however, not significantly different for belimumab-treated patients (anti-Sm: $P=0.0805$, anti-RNP: $P=0.1837$ compared to placebo at week 52). Effects on B-cell subsets over 2 years of treatment were examined in 17 seropositive patients of the initial cohort. ${ }^{46}$ The total number of B cells started to decrease from baseline between days 84 and 168 due to decrease in naive and transitional B cells. CD27+IgD+ memory B cells and plasmablasts decreased only after 532 days, whereas CD27+IgD- memory B cells were not affected, and there were no changes in T cells.

Two open-label, parallel assignment continuation studies (NCT00724867 $7^{47}$ and NCT00712933 ${ }^{48}$ ) of patients completing the BLISS-52 or BLISS-76 trials are ongoing, with projected completion in December 2010, primarily examining belimumab's long-term safety. Additional secondary endpoints in NCT00724867 include disease activity assessments, quality of life measures (SF-36 Health Survey and FACIT-Fatigue scale), and effects on the SLICC/ACR Damage Index, anti-dsDNA and serum complement levels, prednisone use, proteinuria, serum immunoglobulin G, and B-cell subsets. In both studies, belimumab is administered in an open-label, uncontrolled fashion as 1 or $10 \mathrm{mg} / \mathrm{kg}$ IV infusion every 28 days. Furthermore, a phase II multicenter, randomized, open-label trial of subcutaneously administered belimumab in SLE patients is underway (NCT00732940). ${ }^{49}$ Two dosing schedules of belimumab, $100 \mathrm{mg}$ every other week or $100 \mathrm{mg}$ three times 
a week, administered for 6 months are examined. Primary endpoints include adverse events and changes from baseline in B cells and B-cell subsets at weeks 12 and 24. Secondary endpoints include belimumab concentrations following subcutaneous administration and changes from baseline at weeks 12 and 24 in PGA score, SS, serum immunoglobulins, C3/C4 levels, anti-dsDNA levels, and lipid profile.

\section{Conclusions}

Belimumab, a BLyS-specific inhibitor, has shown encouraging results in large RCTs in SLE. Completion of the primary and several secondary endpoints for both belimumab doses in BLISS-52 and the smaller, but still significant, effects observed in BLISS-76 certainly represent major accomplishments. Amidst a limited range of therapeutic options for a disease as complex as SLE, very few of which are FDA approved, the pending FDA review on belimumab is eagerly anticipated.

Belimumab may possibly be the first biologic to be approved in SLE. Further analysis of the BLISS trials and mechanistic studies in the patients enrolled should be encouraged to define its best use. Furthermore, critical review of the BLISS trials, as well as some of the negative SLE trials so far, may help provide insight into efficient SLE trial design that will set the stage for more effective therapies of this disease.

\section{Disclosure}

The authors report no conflicts of interest in this work.

\section{References}

1. Merrill JT. Clinical trials for lupus - are we there yet? Bull NYU Hosp Jt Dis. 2009;67(3):267-270.

2. Merrill JT. What was wrong and might now go right with clinical trials for lupus? Curr Rheumatol Rep. 2009;11(4):235-237.

3. Merrill JT, Buyon JP. Rituximab: wanted dead or alive ... . Arthritis Rheum. 2010;62(8):2188-2191.

4. Baker KP, Edwards BM, Main SH, et al. Generation and characterization of LymphoStat-B, a human monoclonal antibody that antagonizes the bioactivities of B lymphocyte stimulator. Arthritis Rheum. 2003; 48(11):3253-3265.

5. Dörner T, Jacobi AM, Lee J, Lipsky PE. Abnormalities of B cell subsets in patients with systemic lupus erythematosus. J Immunol Methods. 2011;363(2):187-197.

6. Fairhurst AM, Wandstrat AE, Wakeland EK. Systemic lupus erythematosus: multiple immunological phenotypes in a complex genetic disease. Adv Immunol. 2006;92:1-69.

7. Ramanujam M, Davidson A. BAFF blockade for systemic lupus erythematosus: will the promise be fulfilled? Immunol Rev. 2008; 223:156-174.

8. Treml JF, Hao Y, Stadanlick JE, Cancro MP. The BLyS family: toward a molecular understanding of B cell homeostasis. Cell Biochem Biophys. 2009;53(1):1-16.

9. Mackay F, Schneider P. Cracking the BAFF code. Nat Rev Immunol. 2009;9(7):491-502.
10. Khare SD, Sarosi I, Xia XZ, et al. Severe B cell hyperplasia and autoimmune disease in TALL-1 transgenic mice. Proc Natl Acad Sci U S A. 2000;97(7):3370-3375.

11. Ramanujam M, Wang X, Huang W, et al. Similarities and differences between selective and nonselective BAFF blockade in murine SLE. J Clin Invest. 2006;116(3):724-734.

12. Kahn P, Ramanujam M, Bethunaickan R, et al. Prevention of murine antiphospholipid syndrome by BAFF blockade. Arthritis Rheum. 2008; 58(9):2824-2834.

13. Ramanujam M, Bethunaickan R, Huang W, Tao H, Madaio MP, Davidson A. Selective blockade of BAFF for the prevention and treatment of systemic lupus erythematosus nephritis in NZM2410 mice. Arthritis Rheum. 2010;62(5):1457-1468.

14. Stohl W, Jacob N, Guo S, Morel L. Constitutive overexpression of BAFF in autoimmune-resistant mice drives only some aspects of systemic lupus erythematosus-like autoimmunity. Arthritis Rheum. 2010;62(8):2432-2442.

15. Halpern WG, Lappin P, Zanardi T, et al. Chronic administration of belimumab, a BLyS antagonist, decreases tissue and peripheral blood B-lymphocyte populations in cynomolgus monkeys: pharmacokinetic, pharmacodynamic, and toxicologic effects. Toxicol Sci. 2006; 91(2):586-599.

16. Cheema GS, Roschke V, Hilbert DM, Stohl W. Elevated serum B lymphocyte stimulator levels in patients with systemic immune-based rheumatic diseases. Arthritis Rheum. 2001;44(6):1313-1319.

17. Stohl W, Metyas S, Tan SM, et al. B lymphocyte stimulator overexpression in patients with systemic lupus erythematosus: longitudinal observations. Arthritis Rheum. 2003;48(12):3475-3486.

18. Petri M, Stohl W, Chatham W, et al. Association of plasma B lymphocyte stimulator levels and disease activity in systemic lupus erythematosus. Arthritis Rheum. 2008;58(8):2453-2459.

19. Collins CE, Gavin AL, Migone TS, Hilbert DM, Nemazee D, Stohl W. B lymphocyte stimulator (BLyS) isoforms in systemic lupus erythematosus: disease activity correlates better with blood leukocyte BLyS mRNA levels than with plasma BLyS protein levels. Arthritis Res Ther. 2006;8(1):R6.

20. Furie R, Stohl W, Ginzler EM, et al. Biologic activity and safety of belimumab, a neutralizing anti-B-lymphocyte stimulator (BLyS) monoclonal antibody: a phase I trial in patients with systemic lupus erythematosus. Arthritis Res Ther. 2008;10(5):R109.

21. Wallace DJ, Stohl W, Furie RA, et al. A phase II, randomized, double-blind, placebo-controlled, dose-ranging study of belimumab in patients with active systemic lupus erythematosus. Arthritis Rheum. 2009;61(9):1168-1178.

22. Petri M, Wallace DJ, Stohl W, et al. SLE patients with active production of anti-nuclear autoantibodies (ANA) have distinct patterns of lupus activity and peripheral B-cell biomarkers compared to ANA negative patients. Ann Rheum Dis. 2006;65 Suppl 2:356.

23. Furie RA, Petri MA, Wallace DJ, et al. Novel evidence-based systemic lupus erythematosus responder index. Arthritis Rheum. 2009; 61(9):1143-1151.

24. Griffiths B, Mosca M, Gordon C. Assessment of patients with systemic lupus erythematosus and the use of lupus disease activity indices. Best Pract Res Clin Rheumatol. 2005;19(5):685-708.

25. Guidance for Industry: Systemic Lupus Erythematosus - Developing Medical Products for Treatment. Food and Drug Administration; 2006. Available from: http://www.fda.gov/downloads/Drugs/GuidanceComplianceRegulatoryInformation/Guidances/ucm072063.pdf. Accessed November 10, 2010.

26. BENLYSTA ${ }^{\circledR}$ (belimumab). Human Genome Sciences. Available from: http://www.hgsi.com/belimumab.html. Accessed November 10, 2010.

27. Phase 3, Multi-Center, Randomized, Double-Blind, Placebo-Controlled, 52-Wk Study to Evaluate the Efficacy and Safety of Belimumab (HGS1006, LymphoStat-B ${ }^{\mathrm{TM}}$ ), a Fully Human Monoclonal Anti-BLyS Antibody, in Subjects with Systemic Lupus Erythematosus (SLE). NCT00424476. Available from: http://www.clinicaltrials.gov/ct2/show/study/NCT0042447 6 ?term=belimumab\&cond=systemic+lupus+erythematosus\&rank=1 . Accessed November 10, 2010. 
28. A Phase 3, Multi-Center, Randomized, Double-Blind, Placebo-Controlled, 76-Week Study to Evaluate the Efficacy and Safety of Belimumab (HGS1006, LymphoStat-B ${ }^{\text {TM}}$ ), a Fully Human Monoclonal Anti-BLyS Antibody, in Subjects With Systemic Lupus Erythematosus (SLE). NCT00410384. Available from: http://www.clinicaltrials.gov/ct2/show/ NCT00410384?term=belimumab\&cond=systemic+lupus+erythemato sus\&rank=2. Accessed November 10, 2010.

29. Manzi S, Sanchez-Guerrero J, Merrill JT, et al. Belimumab, a BLyS-specific inhibitor, reduced disease activity across multiple organ domains: combined efficacy results from the phase 3 BLISS-52 and -76 studies. Arthritis Rheum. 2010;62 Suppl 10:607.

30. Tanasescu C, Gallacher A, Garcia M, et al. Belimumab, a BLyS-specific inhibitor, significantly improved physical functioning, fatigue, and other health-related quality of life (HRQOL) measures in patients with seropositive systemic lupus erythematosus (SLE): BLISS-52 study. Ann Rheum Dis. 2010;69 Suppl 3:556.

31. Navarra S, Ilianova E, Bae SC, et al. Belimumab, a BLyS-specific inhibitor, reduced disease activity, flares, and steroid use in patients with seropositive systemic lupus erythematosus (SLE): BLISS-52 study. Ann Rheum Dis. 2010;69 Suppl 3:555.

32. Van Vollenhoven R, Zamani O, Wallace DJ, et al. Belimumab, a BLyS-specific inhibitor, reduces disease activity and severe flares in seropositive SLE patients: BLISS-76 study. Ann Rheum Dis. 2010;69 Suppl 3:74.

33. Petri M, Levy RA, Merrill JT, et al. Belimumab, a BLyS-specific inhibitor, reduced disease activity, flares, and prednisone use in patients with seropositive SLE: combined efficacy results from the phase 3 BLISS-52 and -76 studies. Arthritis Rheum. 2010;62 Suppl 10:190.

34. Furie RA, Zamani O, Wallace DJ, et al. Belimumab, a BLyS-specific inhibitor, reduced disease activity and severe flares in seropositive SLE patients: BLISS-76 study results through Wk 76. Arthritis Rheum. 2010;62 Suppl 10:606

35. Guillemin F. Primer: the fallacy of subgroup analysis. Nat Clin Pract Rheumatol. 2007;3(7):407-413.

36. Bertsias GK, Ioannidis JP, Boletis J, et al. EULAR points to consider for conducting clinical trials in systemic lupus erythematosus: literature based evidence for the selection of endpoints. Ann Rheum Dis. 2009;68(4):477-483.

37. Kiani AN, Petri M. Quality-of-life measurements versus disease activity in systemic lupus erythematosus. Curr Rheumatol Rep. 2010;12(4):250-258.

38. Strand V, Levy RA, Cervera R, et al. Belimumab, a BLyS-specific inhibitor, improved fatigue and SF-36 physical and mental component summary scores in patients with SLE: BLISS-76 and -52 studies. Arthritis Rheum. 2010;62 Suppl 10:773.

39. Levy RA, Ilianova E, Ionescu R, et al. Belimumab, a BLyS-specific inhibitor, significantly reduced autoantibodies and normalized low complement in patients with seropositive systemic lupus erythematosus (SLE): BLISS-52 study. Ann Rheum Dis. 2010;69 Suppl 3:556.
40. Stohl W, Hiepe F, Thomas M, et al. Belimumab, a BLyS-specific inhibitor, significantly reduced autoantibodies, normalized low complement, and reduced selected B-cell populations in patients with seropositive systemic lupus erythematosus (SLE): the phase 3 BLISS studies. Arthritis Rheum. 2010;62 Suppl 10:480.

41. Chatham W, Wallace DJ, Stohl W, et al. Effect of belimumab, a B-lymphocyte stimulator-specific inhibitor, on functional antibodies to pneumococcal, tetanus, and influenza vaccines. Arthritis Rheum. 2010; 62 Suppl 10:192.

42. Chatham W, Weinstein A, Petri M, et al. Five-year safety and efficacy experience with belimumab, a BLyS-specific inhibitor, in patients with systemic lupus erythematosus (SLE). Ann Rheum Dis. 2010; 69 Suppl 3:147.

43. Merrill JT, Wallace DJ, Furie RA, et al. Five-year experience with belimumab, a BLyS-specific inhibitor, in patients with systemic lupus erythematosus (SLE). Arthritis Rheum. 2010;62 Suppl 10:608.

44. Ginzler EM, Wallace DJ, Chatham W, et al. Belimumab (fully human monoclonal antibody to BLyS) reduced steroid use in systemic lupus erythematosus (SLE) patients during 3 years of therapy. Ann Rheum Dis. 2008;67 Suppl 2:217.

45. Chatham W, Aranow C, Furie R, et al. Progressive normalization of autoantibody, immunoglobulin, and complement levels over 3 years of belimumab (fully human monoclonal antibody to BLyS) therapy in systemic lupus erythematosus (SLE) patients. Ann Rheum Dis. 2008; 67 Suppl 2:217.

46. Jacobi AM, Huang W, Wang T, et al. Effect of long-term belimumab treatment on B cells in systemic lupus erythematosus: extension of a phase II, double-blind, placebo-controlled, dose-ranging study. Arthritis Rheum. 2010;62(1):201-210.

47. A Multi-Center, Continuation Trial of Belimumab (HGS1006, LymphoStat-B ${ }^{\mathrm{TM}}$ ), a Fully Human Monoclonal Anti-BLyS Antibody, in Subjects With Systemic Lupus Erythematosus (SLE) Who Completed the Phase 3 Protocol HGS1006-C1056 in the United States. NCT00724867. Available from: http://www.clinicaltrials.gov/ct2/show/NCT00724867? term=belimumab\&rank=9. Accessed November 10, 2010.

48. A Multi-Center, Continuation Trial of Belimumab (HGS1006, LymphoStat-B ${ }^{\mathrm{TM}}$ ) a Fully Human Monoclonal Anti-BLyS Antibody in Subjects With Systemic Lupus Erythematosus (SLE) Who Completed the Phase 3 Protocol HGS1006-C1056 or HGS1006-C1057. NCT00712933. Available from: http://www.clinicaltrials.gov/ct2/show/NCT00712933? term=belimumab\&rank=11. Accessed November 10, 2010.

49. A Phase 2, Multi-Center, Randomized, Open Label, Trial to Evaluate the Safety, Tolerability, and Biological Activity of 2 Dosing Schedules of Belimumab (HGS1006, LymphoStat-B ${ }^{\mathrm{TM}}$ ), a Fully Human Monoclonal Anti-BLyS Antibody, Administered Subcutaneously to Subjects With Systemic Lupus Erythematosus (SLE). NCT00732940. Available from: http://www.clinicaltrials.gov/ct2/show/study/NCT00732940?term= belimumab\&cond=systemic+lupus+erythematosus\&rank=3. Accessed November 10, 2010.
Biologics: Targets \& Therapy

\section{Publish your work in this journal}

Biologics: Targets \& Therapy is an international, peer-reviewed journal focusing on the patho-physiological rationale for and clinical application of Biologic agents in the management of autoimmune diseases, cancers or other pathologies where a molecular target can be identified. This journal is indexed on PubMed Central, CAS, EMBase, Scopus

\section{Dovepress}

and the Elsevier Bibliographic databases. The manuscript management system is completely online and includes a very quick and fair peerreview system, which is all easy to use. Visit http://www.dovepress. com/testimonials.php to read real quotes from published authors. 